\title{
Ineffective Feeding Practices and Their Effect on Malocclusion: A Narrative Review
}

\author{
Pratima R. Mokashi ${ }^{1} \quad$ Srikala Bhandary ${ }^{1}$ \\ ${ }^{1}$ Department of Pediatric and Preventive Dentistry, A.B. Shetty \\ Memorial Institute of Dental Sciences, Nitte (deemed to be) \\ University, Mangaluru, Karnataka, India
}

\begin{abstract}
Address for correspondence Srikala Bhandary, BDS, MDS, Reader Department of Pediatric and Preventive Dentistry, A.B. Shetty Memorial Institute of Dental Sciences, Nitte (deemed to be) University, Mangaluru, Karnataka 575018, India (e-mail: drsrikalabhandary@nitte.edu.).
\end{abstract}

\begin{abstract}
Keywords

- breastfeeding

- bottle feeding

- ineffective breastfeeding

- malocclusion

- pacifier

Objective The aim of this narrative review is to highlight the association of ineffective feeding practices with the development of malocclusion in children and the role of a pediatric dentist in identifying the cues and signs of improper feeds, and encourage effective breastfeeding practices.

Introduction There has been an ongoing debate on the role of effective breastfeeding in the prevention of malocclusion. Although no specific claim supports the positive impact of the same, a detailed reviewing of the literature helps to identify the method of feeding to be chosen considering health benefits and personal preference.

Materials and Methods A broad search of all resources linked to the topic was performed in PubMed, Medline, World Health Organization web site, government web sites, and Google Scholar search engine. Keywords used in the search included breastfeeding, ineffective breastfeeding, bottle feeding, pacifier, and malocclusion. A total of 60 articles published in the period from 2000 to 2019 were segregated. Selected articles comprised original research, meta-analysis, and systematic reviews.

Results Parameters such as duration, posture, and non-nutritive sucking habits had an impact on effective breastfeeding.

Discussion Effective breastfeeding and maintaining appropriate posture and duration helps to positively impact the normal growth and development of the jaws, muscular functioning, and speech. Thereby, the risk of developing malocclusion in primary dentition can be prevented.

Conclusion An understanding of the role of breastfeeding and malocclusion will help in the early intervention and prevention of malocclusion and deviated muscular function. Highlighting the role of counseling and effective feeding practices is also an area that should be focused upon by budding clinicians.
\end{abstract}

\section{Introduction}

\section{Understanding Breast Feeding}

Breastfeeding is a quintessential and an unpressed way of providing food for the physiologic growth and the development of infants. ${ }^{1}$ Review of evidence has displayed that an ideal practice for breastfeeding should be solely for 6 months. ${ }^{2}$ Thereafter supplementary foods with continued breastfeeding for up to 2 years of age may be resumed. ${ }^{2}$ To enable mothers to authorize and sustain published online

July 23, 2021
DOI https://doi.org/

$10.1055 / \mathrm{s}-0041-1731912$

ISSN 2582-4287 (c) 2021. Nitte (Deemed to be University).

This is an open access article published by Thieme under the terms of the Creative Commons Attribution-NonDerivative-NonCommercial-License, permitting copying and reproduction so long as the original work is given appropriate credit. Contents may not be used for commercial purposes, or adapted, remixed, transformed or built upon. (https://creativecommons.org/licenses/by-nc-nd/4.0/).

Thieme Medical and Scientific Publishers Pvt. Ltd. A-12, 2nd Floor, Sector 2, Noida-201301 UP, India 
exclusive breastfeeding for 6 months, the World Health Organization (WHO) and United Nations Children's Fund (UNICEF) have recommended the commencement of breastfeeding within the first hour of life and persuasions of exclusive breastfeeds up to 6 months. It discourages the use of pacifiers, bottles, or teats (-Table $\mathbf{1}$ ).

Breastfeeding helps to prevent malocclusion. Breastfeeding has a very crucial role in development of craniofacial region as it directly has an impact on the orofacial musculature ${ }^{3}$ (-Table 2). As a result of which it enhances and supports the sagittal growth and advancement in the intermaxillary relationship, thus preventing or reducing the incidences of malocclusion. This interconnection between breastfeeding and malocclusion can be directly linked to that of prevention of acquisition of non-nutritive sucking habit (NNSH). ${ }^{4}$

There are three factors in breastfeeding which have direct implications on the development of the orofacial structure, and the primary and the subsequent permanent denti$\operatorname{tion}^{4}$ (-Fig. 1).

There has been an ongoing debate in the role of effective feeding in the prevention of malocclusion. Although no specific claim supports the positive impact of the same.

Table 1 General benefits of breastfeeding

\section{General health benefits of breastfeeding}

- Aids in the growth and development of infant. ${ }^{1}$

- Aids in the growth and development of infant. ${ }^{1}$

- Immunological protection to the child. ${ }^{1}$

- Immunological protection to the child. ${ }^{1}$

- Maturation of gastric microflora. ${ }^{1}$

- Maturation of gastric microflora. ${ }^{1}$

- Protection against otitis media as opposed to use of pacifiers. ${ }^{1}$

- Protection against otitis media as opposed to use of pacifiers. ${ }^{1}$

- Prevention of gastrointestinal disorders. ${ }^{1}$

- Prevention of gastrointestinal disorders. ${ }^{1}$

- Protection against diabetes and long-term obesity. ${ }^{1}$

- Protection against diabetes and long-term obesity. ${ }^{1}$

- Decreases the risk of ovarian cancers in mothers. ${ }^{1}$

- Decreases the risk of ovarian cancers in mothers. ${ }^{1}$

Table 2 Effect of breastfeeding on the development of occlusion

Occlusal benefits of breastfeeding
- Biologic growth and development of the maxilla and
mandible. ${ }^{4}$
- Biologic growth and development of the maxilla and
mandible. ${ }^{4}$
- Appropriate shaping of hard palate and arch forms. ${ }^{4}$
- Appropriate shaping of hard palate and arch forms. ${ }^{4}$
- Induce normal sucking patterns and maintain normal
- - Induce position. ${ }^{4}$
- Enormal sucking patterns and maintain normal
- Elimination of development of posterior crossbites. ${ }^{4}$
- Elimination of development of posterior crossbites. ${ }^{4}$
- Elimination of development of anterior open bite. ${ }^{4}$

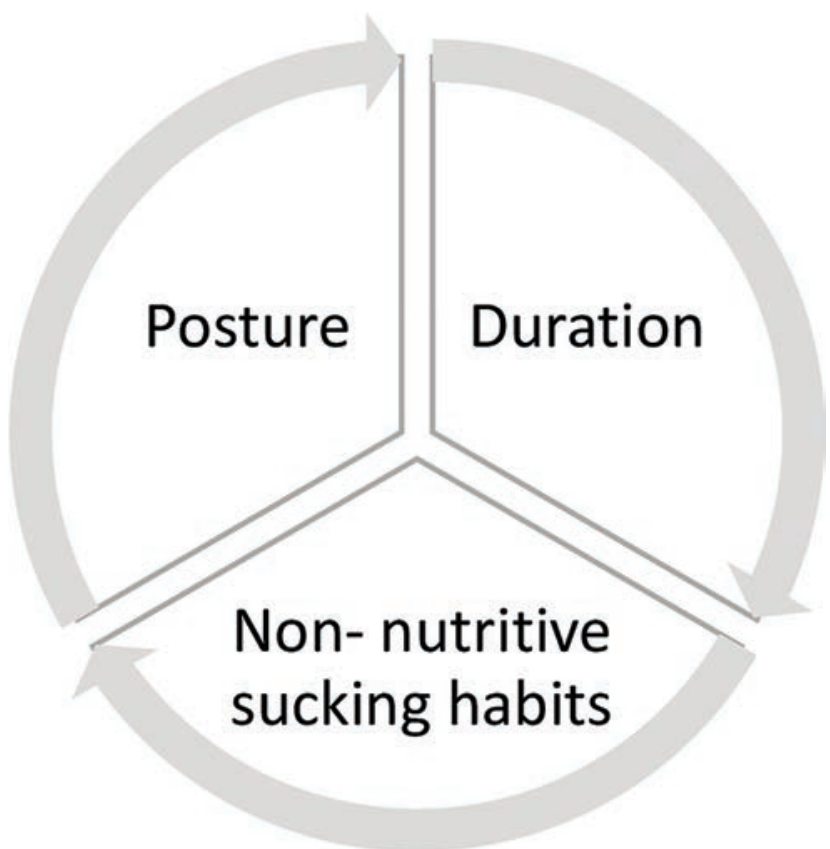

Fig. 1 Factors influencing development of orofacial structure.

\section{Objective}

The aim of this narrative review is to highlight the association of ineffective feeding practices with the development of malocclusion in children and the role of a pediatric dentist in identifying the cues and signs of improper feeds and encourage effective breastfeeding (EBF) practices.

\section{Materials and Methods}

A broad search of all resources linked to the topic was performed in PubMed, Medline, World Health Organization web site, government web sites, and Google Scholar search engine. Keywords used in the search included breastfeeding, ineffective breastfeeding, bottle feeding, pacifier, and malocclusion. A total of 60 articles published in the period from 2000 to 2019 were segregated. Selected articles comprised original research, meta-analysis, and systematic reviews. Articles excluded comprised unavailability of the full version.

\section{Role of Duration and Posture of Breastfeeding and Its Impact on Malocclusion}

Muscular activity has a predominant role in the infant's survival. The activity of the cheeks and the tongue in a new born infant not only aids in the development of occlusion but also in the growth of the jaws. ${ }^{4,5}$ The improvement of occlusion largely depends on the tensional forces created during different orofacial functions. The initial phase of postnatal life thereby plays an utmost important role. Malocclusion seems to be linked to an imbalance of the functional forces of the oral and facial musculature. . $^{4}$

It was thereby noted that breastfeeding, if lengthened, tends to exert a protective response against malocclusion. The primary factor being that the muscles which contribute 
to feeding activity may either be impaired (orbicularis oris, masseter), hyperactive (chin muscles), or malpositioned (tongue is pushed backward) and may produce a crippled dentofacial development of the child. Children who are not breastfed for prolonged durations are more prone to develop inappropriate muscular tonicity in comparison to the ones breastfed for longer duration. Similarly early refrainment from breastfeeding practices and indulgence in bottle feeding may also be a contributory factor for the same. ${ }^{7}$

In addition to the duration, appropriate positioning of the baby's body is important for good attachment and successful feeding. The process of breastfeeding can be acclaimed to be effective only if there is an adequate milk flow to prevent any feeding problems. ${ }^{7}$ EBF can be defined as an appropriate positioning, latching, and sucking behavior of the baby to the breasts. ${ }^{7}$ Although EBF is a biologic phenomenon, many factors need to be taken into consideration for effective implementation. Semireclined or lying down postures in the child could lead to interference in the adequate flow of milk supply to the child. This may lead to nipple sucking instead of suckling mechanism. This leads to increased incidences of muscular fatigue in the child 7 . Signs suggesting poor positioning are sucked in cheeks, lips not flanged out and back, noisy feeding, space between breast and chin, constant or prolonged feeding, and multiple jaw movements in attempts to thrive without periods per swallow. ${ }^{8}$ Thus, there is a considerable lack of satiety that arises for the infant. This leads to undue tiredness since the infant has to exert considerable amount of muscular forces to prevent the nipple from slipping out of the mouth. ${ }^{9}$

Four key points about positioning a baby are as follows:

- Baby's head and body should be placed in a straight line.

- Baby should be held close to mother's body.

- Baby's whole body should be adequately supported.

- Baby approaches breast, nose to nipple.

\section{Use of Pacifiers and Bottle Feeding and Its Role in Malocclusion}

Non-nutritive sucking (NNS) is characterized as the use of muscular activity for the purpose of swallowing, except when fluid is not introduced. Nutritive suckling (NS) is wherein there is use of a series of muscular activity following introduction of fluid, leading to bolus formation in the mouth and throat. ${ }^{10-12}$ It was observed by Wolff that the sucking frequency and pressure decreased, while the duration of sucking increased in NS. In case of breastfeeding there is a shift from NNS to NS that takes place. ${ }^{13}$

Pacifiers are commonly used to comfort and distract crying babies. This aids to quench the child's instinctive need to satisfy the oral stage. Pacifiers also serve as an alternative to curb habits such as thumb sucking. ${ }^{14}$ As pacifiers are a form of NNS, their use may be a hazardous factor for the premature curbing of EBF. ${ }^{10}$ The use of pacifiers may be linked with "nipple confusion," early weaning, otitis media, gastrointestinal infections, and malocclusions.

The recommended age to halt the use of pacifier has been suggested to be 2 years. The continued use of pacifiers for 4 or more years may be considered to be a lengthy habit. ${ }^{15}$ The three factors that are associated with the relationship of malocclusion and pacifier use are the intensity, the frequency, and the length of pacifier use. ${ }^{15}$ The number of hours of usage of a pacifier also plays a crucial role. Warren et al have suggested the use of pacifiers for not more than 4 to 6 hours. The ideal time for usage is when the infant is asleep. ${ }^{16}$ The characteristic features that accompany the extended use of pacifiers are constriction of the superior dental arch, especially at the area of the canine teeth, along with the enlargement of the inferior dental arch. ${ }^{17}$

Due to the constant sucking action, the tongue is in a lower position in the oral cavity ${ }^{15}$. Posterior crossbite, anterior open-bite, and increased overjet, class II relationship with respect to molar and canine are the most common malocclusions encountered due to extended pacifier use. In addition to the skeletal and dental deformities, the continuous use of pacifier can lead to several myofunctional impairments, such as labial incompetence, lip entrapment, and decreased muscular tonicity. ${ }^{15-17}$

It has been suggested that a pacifier may decrease oral sugar clearance, in a similar way as removable dentures do, which would prolong conditions of low $\mathrm{pH}$ in plaque and favor the selection of aciduric microorganisms. It also aids to increase the available surface for microbial adhesion. ${ }^{18}$

Bottle feeds limit the potential use of the perioral muscles and thereby act as a primary agent in the etiology of malocclusion. ${ }^{11}$ The process of sucking differs between children who are breastfed and those who are fed from a bottle. Children who are breastfed present greater facial muscle activity than those who are bottle-fed, thus promoting more adequate craniofacial growth and development of jaw bones. The movement of lips and tongue during breastfeeding forces the child to draw breast milk through a squeeze action, while for children who are bottle-fed the movement for obtaining the milk is more passive; therefore, there is greater potential to develop a malocclusion. ${ }^{19}$

The brachycephalic mandibular arch format is more easily reached when the child is breastfed, which in turn allows appropriate tooth eruption. ${ }^{19}$ Another aspect of anatomy in favor of breastfeeding is that the mother's nipple adapts to the internal shape of the oral cavity, enabling a perfect oral seal, which in turn leads to satisfactory development of nasal breathing. It is well acknowledged that children who have nasal breathing are less likely to develop open-mouth posture, which in turn may result in an excessive vertical facial dimension. ${ }^{19}$

\section{Common Malocclusions in Relation to Feeding Practices}

Malocclusion is a morphological variation which may or may not be associated with underlying pathological condition. It is regarded as an umbrella term which is a group of developmental disorders arising from varied causes and not a single etiologic factor. ${ }^{18}$ Although genetic factors also play an important role in the development of malocclusion, it is important to understand that the supporting perioral forces and deleterious habits also negatively impact the arch development. ${ }^{19}$ 


\section{Prevalence of Anterior Open Bite in Primary Dentition}

Subtelney and Sakuda have defined anterior open bite as an open vertical dimension between the incisal edges of the maxillary and mandibular anterior teeth, although loss of vertical dental contact can occur between the anterior or the buccal segment. ${ }^{20}$ This deviation in the incisor relationship in younger children (transition from the primary to the mixed dentition phases) is associated with NNSHs and is not self-correcting anomaly. ${ }^{20}$ By the time the child reaches adolescence, the environmental causes become less significant than the skeletal causes. Johnson and Larson used the term non-nutritive sucking to describe habits that involve digits, pacifiers, and other environmental influences. Children who are exclusively bottle-fed or those who are introduced to artificial feeding in the first 3 months of life are presented with anterior open bite. The causation factor here being that bottle feeding tends to cause atypical swallowing pattern in these children having harmful influences on developing occlusion. ${ }^{20}$ Lack of feed tends to promote the use of pacifier and digit sucking habits which are non-nutritive in nature and these tend to be continued until 4 to 5 years of age. ${ }^{21}$ The link extended use of pacifier and habits can be explained on the basis of Freud's psychoanalytical and learning theory. As per this theory all children possess a built in drive for sucking in conjugation with the rooting and attachment reflexes. The various environmental factors such as early weaning, cessation of breastfeeding, and introduction of the use of pacifiers contribute to the transfer of this sucking drive to non-nutritive sources, such as pacifiers, thumb, and fingers.

It is characterized by asymmetric anterior open bite and a transverse constriction of the maxillary arch. An increased overjet (horizontal projection of maxillary teeth beyond the mandibular anterior teeth) is strongly related to NNSHs and their presence predisposes the child to an increased risk of traumatic dental injury. Such incisor relationships that shift from normal tend to evoke undesirable social responses. ${ }^{21}$ The hyperdivergent facial pattern are more prone to anterior open bite. Thus, prolonged sucking habits and hyperdivergent facial patterns are a significant risk factor for anterior open bite. As the NNSH increases in the child, there is a 2.38 times higher chance of developing an anterior open bite. ${ }^{20}$

Children who were breastfed for less than 6 months would be 9.3 times more prone to develop an open bite than those who were fed for extended duration. Thus, breastfeeding plays a protective role in the prevention of deviation of normal occlusion..$^{22}$ Thus, the protective nature of breastfeeding not only helps in reducing the acquisitions of NNSHs but also the development of anterior open bite in the developing dentitions. ${ }^{23}$

Prevalence of Posterior Cross Bite in Primary Dentition Posterior crossbite is one of the most common malocclusion encountered during the deciduous dentition phase. Absence of any early intervention leads to facial asymmetry and temporomandibular disorders. Along with deviation in occlusion, posterior crossbite also has a debilitating effect on craniofacial growth and development as a result of the adverse influence on the muscles. This hyperactivity of muscles leads to an impairment of facial aesthetics. ${ }^{24}$

It was proclaimed by many authors that pacifier sucking was one of the causative factors involved in the formation of posterior crossbite. The decreased intermolar arch width contributing to posterior crossbite is the result of prolonged use of pacifiers. ${ }^{25}$ Early weaning resulted in poor muscular activity as opposed to breastfeeding sucks. ${ }^{26}$ Bottle feeding, even among breastfed children, interfered negatively with orofacial development. ${ }^{26}$ Open-mouth posture, as a habit or as a results of adenotonsillar enlargement or prolonged inflammation of the nasal mucosa related to allergies or chronic infections, inhibits transverse maxillary growth and results in an increase in the prevalence of posterior crossbite. ${ }^{27}$ Mouth breathing is also known to be associated with an increased vertical dimension, which also impairs the transverse dimension. Tongue tie or short lingual frenula leads to low posture of the tongue, thereby being a contributory factor to the development of posterior crossbite. This disturbs the orofacial dynamics between the tongue, the lips, and the cheeks. These dental arch parameters were known to be persisting beyond the cessation of the habit. ${ }^{28}$

\section{Prevention of Malocclusions}

Preventive and interceptive orthodontics has been a subject of continuous dispute with respect to treatment timing, fruitfulness, physiological, and psychological benefits. ${ }^{29}$ For the enhancement of dental and skeletal development, it has been advised to initiate an early intervention. This not only aids to facilitate the correction of habits, improper functions, and malocclusions in their early stages, but also the compromised transverse dimensions that can cause temporo mandibular joint problems or facial asymmetry. ${ }^{29}$

Thus, breastfeeding, although a boon, comes with its checklist of cautions. To minimize the effects of ineffective breastfeeding it is important to raise awareness about EBF. ${ }^{30}$ Tracing the improper postures at an early stage may also help to gain control over the unwanted results of ineffective breastfeeding. Counseling the mother plays a very important role. This should be reinforced during pregnancy and puerperium, including preventive dental visits even before the child's first tooth appears. ${ }^{30}$

As mothers are more actively involved with child care, hence maternal education plays a vital role in the improvement of oral habits. The level of awareness and active participation by the parents in the child's welfare has been linked to their socioeconomic status. ${ }^{30}$ Women need extra support, encouragement, and reassurance while breastfeeding. Although we view breastfeeding as a natural action, it is still a skill that has be learned. Initially breastfeeding can seem demanding, because the baby may have an urge to feed/suck frequently. Babies, however, begin to establish their own pattern over time, and the mother will begin to feel more comfortable and at ease. ${ }^{31}$

Regular dental visits can moderate the negative impacts of low maternal education to pacifier usage, highlighting the critical role of pediatric dentists, particularly among children of mothers with low and medium educational levels. ${ }^{32}$ 
Every clinical examination of a toddler with NNSHs should include assessments of the length of the frenulum linguae and thereby the resulting tongue posture ${ }^{32} \mathrm{~A}$ detailed case history should be taken to analyze any deleterious oral habits, if present. If any deleterious habit has been spotted, immediate intervention by the pediatric dentist should be commissioned. This may include a combination of the patient education and motivation along with interceptive orthodontics. Interceptive orthodontics helps to reduce the risk of deviation from normal anatomy. ${ }^{33}$

\section{Conclusion}

It can be emphasized that educational issues involved in pediatric dentistry, the vital role of dental care practitioners in the promotion of healthy oral habits and the need for identifying specific target groups.

To conclude, exclusive breastfeeding is strongly and inversely associated with the frequency, intensity, and duration of pacifier use, which may lead to severe malocclusion. Thus ineffective breastfeeding has been known to influence the growth patterns in the individual, causing malocclusions and deformities in speech, aesthetics profiles, and function. ${ }^{33}$

\section{Clinical Significance}

The main focus of this article was to highlight the importance of EBF and the role of a pediatric dentist in identifying the early cues and signs of improper feeds. An understanding of the role of breastfeeding and malocclusion will help in the early intervention and prevention of malocclusion and deviated muscular function. Evidence-based recommendations help clinicians to provide the best care and advice to parents on infant feeding guidelines. Once an awareness has been raised about the long-term physiologic and social impact of malocclusion, only then can we pave the way for early interventional orthodontics.

\section{Conflict of Interest}

None declared.

\section{References}

1 Anatolitou F. Human milk benefits and breastfeeding. J of Pedriatic Neonat Individual Med. 2012;1(1):11-18

2 Kramer MS, Kakuma R. The optimal duration of exclusive breastfeeding: a systematic review. Adv Exp Med Biol 2004;554:63-77

3 Sai Sunil Kishore M. Breast feeding knowledge practices amongst mothers in India. J Tropical Pedriatics 2008;2:113

4 Roscoe MG, da Silva Bonifacio SV, da Silva TB, Pingueiro JM, Lemos MM, Feres MF. Association of breastfeeding duration, nonnutritive sucking habits, and malocclusion. Int J Clin Pediatr Dent 2018;11(1):18-22

5 Drewett RF, Woolridge M. Sucking patterns of human babies on the breast. Early Hum Dev 1979;3(4):315-321

6 Ganesh M, Tandon S, Sajida B. Prolonged feeding practice and its effects on developing dentition. J Indian Soc Pedod Prev Dent 2005;23(3):141-145
7 Kronborg H, Vaeth M. How are effective breastfeeding technique and pacifier use related to breastfeeding problems and breastfeeding duration. ? Birth 2009;36(1):34-42

8 Victora CG, Bahl R, Barros AJ, et al. Lancet Breastfeeding Series Group. Breastfeeding in the 21st century: epidemiology, mechanisms, and lifelong effect. Lancet 2016;387(10017):475-490

9 Colson SD, Meek JH, Hawdon JM. Optimal positions for the release of primitive neonatal reflexes stimulating breastfeeding. Early Hum Dev 2008;84(7):441-449

10 Rendón Macías ME, Meneses GJ. Physiology of nutritive sucking in newborns and infants. Bol Méd Hosp Infant México 2011;68(4):319-327

11 Mizuno K, Ueda A. Changes in sucking performance from nonnutritive sucking to nutritive sucking during breast- and bottle-feeding. Pediatr Res 2006;59(5):728-731

12 Lau C, Kusnierczyk I. Quantitative evaluation of infant's nonnutritive and nutritive sucking. Dysphagia 2001;16(1):58-67

13 Woolridge MW. The 'anatomy' of infant sucking. Midwifery 1986;2(4):164-171

14 Chandran L, Gelfer P. Breastfeeding: the essential principles. Pediatr Rev 2006;27(11):409-417

15 Buccini GDS, Pérez-Escamilla R, Venancio SI. Pacifier use and exclusive breastfeeding in Brazil. J Hum Lact 2016;32(3):NP52-NP60

16 Medeiros R, Ximenes M, Massignan C, et al. Malocclusion prevention through the usage of an orthodontic pacifier compared to a conventional pacifier: a systematic review. Eur Arch Paediatr Dent 2018;19(5):287-295

17 Buccini GDS, Pérez-Escamilla R, Paulino LM, Araújo CL, Venancio SI. Pacifier use and interruption of exclusive breastfeeding: systematic review and meta-analysis. Matern Child Nutr 2017;13(3):e12384

18 Pérez-Suárez V, Carrillo-Diaz M, Crego A, Romero M. Maternal education, dental visits and age of pacifier withdrawal: pediatric dentist role in malocclusion prevention. J Clin Pediatr Dent 2013;37(3):315-319

19 Peres KG, Cascaes AM, Nascimento GG, Victora CG. Effect of breastfeeding on malocclusions: a systematic review and meta-analysis. Acta Paediatr 2015;104(467):54-61

20 Romero CC, Scavone-Junior H, Garib DG, Cotrim-Ferreira FA, Ferreira RI. Breastfeeding and non-nutritive sucking patterns related to the prevalence of anterior open bite in primary dentition. J Appl Oral Sci 2011;19(2):161-168

21 Doğramaci EJ, Rossi-Fedele G, Dreyer CW. Effect of breastfeeding on different features of malocclusions in the primary dentition: a systematic review protocol. JBI Database Syst Rev Implement Reports 2017;15(7):1856-1866

22 Bishara SE, Warren JJ, Broffitt B, Levy SM. Changes in the prevalence of nonnutritive sucking patterns in the first 8 years of life. Am J Orthod Dentofacial Orthop 2006;130(1):31-36

23 Cozza P, Baccetti T, Franchi L, Mucedero M, Polimeni A. Sucking habits and facial hyperdivergency as risk factors for anterior open bite in the mixed dentition. Am J Orthod Dentofacial Orthop 2005;128(4):517-519

24 Melink S, Vagner MV, Hocevar-Boltezar I, Ovsenik M. Posterior crossbite in the deciduous dentition period, its relation with sucking habits, irregular orofacial functions, and otolaryngological findings. Am J Orthod Dentofacial Orthop 2010;138(1):32-40

25 Agarwal SS, Sharma M, Nehra K, Jayan B, Poonia A, Bhattal $\mathrm{H}$. Validation of association between breastfeeding duration, facial profile, occlusion, and spacing: a cross-sectional study. Int J Clin Pediatr Dent 2016;9(2):162-166

26 Kobayashi HM, Scavone H Jr, Ferreira RI, Garib DG. Relationship between breastfeeding duration and prevalence of posterior crossbite in the deciduous dentition. Am J Orthod Dentofacial Orthop 2010;137(1):54-58 
27 de Sousa RV, Ribeiro GL, Firmino RT, Martins CC, Granville-Garcia AF, Paiva SM. Prevalence and associated factors for the development of anterior open bite and posterior crossbite in the primary dentition. Braz Dent J 2014;25(4):336-342

28 Limeira AB, Aguiar CM, de Lima Bezerra NS, Câmara AC. Association between breast-feeding duration and posterior crossbites. J Dent Child (Chic) 2014;81(3):122-127

29 Traebert E, Zanini FA, Nunes RD, Traebert J. Nutritional and non-nutritional habits and occurrence of malocclusions in the mixed dentition. An Acad Bras Cienc 2020;92(1):e20190833
30 Thomas JA. Guidelines for bottle feeding your premature baby. Adv Neonatal Care 2007;7(6):311-318

31 Thoyre SM, Shaker CS, Pridham KF. The early feeding skills assessment for preterm infants. Neonatal Netw 2005;24(3):7-16

32 Meier P, Pugh EJ. Breast-feeding behavior of small preterm infants. MCN Am J Matern Child Nurs 1985;10(6):396-401

33 Cordell A, Elverson C. Interventions to improve breastfeeding outcomes from six weeks to six months: a systematic review. West J Nurs Res 2021;43(6):583-596 